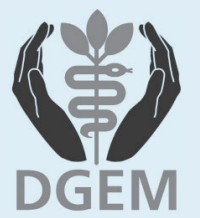

\title{
S3-Leitlinie der Deutschen Gesellschaft für Ernährungsmedizin (DGEM)
} in Zusammenarbeit mit der GESKES, der AKE und der DGVS Klinische Ernährung in der Gastroenterologie (Teil 2) - Pankreas

\author{
S3-Guideline of the German Society for Nutritional Medicine (DGEM) in Cooperation \\ with the GESKES, the AKE and the DGVS \\ Clinical Nutrition in the Gastroenterology (Part 2) - Pancreas
}

Autoren

Institute
J. Ockenga' ${ }^{1}$, Chr. Löser ${ }^{2}$, M. Kraft ${ }^{3}$, C. Madl ${ }^{4}$ und das DGEM Steering Committee*

Die Institute sind am Ende des Artikels gelistet.

\author{
Schlüsselwörter \\ - akute Pankreatitis \\ - chronische Pankreatitis \\ - Ernährung \\ - enterale Ernährung \\ - parenterale Ernährung \\ - Diät \\ - Leitlinie \\ Keywords \\ - acute pancreatitis \\ - chronic pancreatitis \\ - nutrition \\ - enteral feeding \\ - parenteral feeding \\ - diet \\ - guideline
}

\section{Bibliografie}

Dol http://dx.doi.org/ 10.1055/s-0033-1360014

Aktuel Ernahrungsmed 2014;

39: e43-e56

(c) Georg Thieme Verlag KG

Stuttgart · New York

ISSN 0341-0501

\section{Korrespondenzadresse}

Prof. Dr. J. Ockenga

Klinikum Bremen Mitte, Medizinische Klinik II -

Gastroenterologie,

Endokrinologie \&

Ernährungsmedizin

St.-Jürgen-Straße 1

28177 Bremen

Tel.: +49 (0)421 4975354

johann.ockenga@klinikum-

bremen-mitte.de

\section{Zusammenfassung \\ $\nabla$}

Fragestellung: Die akute und chronische Pankreatitis sind häufige Erkrankungen. Während ca. $80 \%$ der Patienten mit akuter Pankreatitis eine eher geringe Morbidität aufweisen, verläuft die Erkrankung bei $20 \%$ der Patienten schwer mit z.T. signifikanter Letalität. Die chronische Pankreatitis hat aufgrund der oftmals schmerzbedingten reduzierten Nahrungsaufnahme und einer Maldigestion ein hohes Risiko für Ernährungsdefizite. Die vorliegende S3-Leitlinie gibt Empfehlungen zum ernährungsmedizinischen Management dieser Erkrankungen.

Methodik: Es wurde eine systematische Literaturrecherche zur Ernährung bei den genannten Krankheitsbildern durchgeführt. Die Ergebnisse wurden in einer interdisziplinären Arbeitsgruppe diskutiert. Auf dieser Basis wurden von der Arbeitsgruppe 32 Empfehlungen erarbeitet und bewertet, die im Rahmen der vorliegenden S3-Leitlinie in einem Delphi-Verfahren und einer Konsensuskonferenz vorgestellt, diskutiert und verabschiedet wurden.

Ergebnisse: In der ernährungsmedizinischen Betreuung von Patienten mit akuter Pankreatitis hat es in den letzten 15 Jahren einen wesentlichen Paradigmenwechsel gegeben. Gestützt auf entsprechende Studien können mit signifikanter Evidenz Empfehlungen für die Ernährung von Patienten mit akuter Pankreatitis gegeben werden. Während bei der leichten bis moderaten akuten Pankreatitis eine liberale und frühzeitige orale Nahrungsaufnahme möglich ist, ist bei der schweren akuten Pankreatitis eine frühzeitige enterale künstliche Ernährung indiziert. Durch diese Maßnahme kann nicht nur die Morbidität, sondern auch die Letalität gesenkt werden. Patienten mit chronischer Pankreatitis profitieren von einem ernährungsmedizinischen Assessment und gezielten diätetischen Interventionen.

\section{Abstract \\ $\nabla$}

Purpose: Acute and chronic pancreatitis are frequent diseases with relevant clinical impact. About $80 \%$ of patients with an acute pancreatitis have a mild or moderate course of the disease. However, $20 \%$ develop a severe life threatening disease. Patients with chronic pancreatitis often have an increased risk for malnutrition due to feeding associated pain or maldigestion. The current S3 guideline gives evidence-based recommendations for adequate nutritional support due to the individualized course of disease.

Methods: A systematic literature search about nutrition and the mentioned disease patterns was conducted. The results were discussed in an interdisciplinary working group. This group developed 32 recommendations, which were presented, discussed and confirmed in a consensus conference. Results: In the last 15 years major changes in the nutritional support of patients with pancreatitis have been developed. The current S3 guideline provides evidence based recommendations for the indications, application and type of nutritional support to be used in acute and chronic phases of pancreatic disease. In patients with mild/moderate acute pancreatitis oral feeding should be started liberal. In patients with severe acute pancreatitis an early enteral nutrition has been shown to significantly reduce morbidity and mortality. In chronic pancreatitis nutritional assessment and support are mandatory.

Conclusion: An individualized nutritional support is an important part of the multimodal therapy of patients with acute or chronic pancreatitis.

\footnotetext{
* DGEM Steering Committee: Bischoff SC, Lochs H, Weimann A sowie das DGEM-Präsidium
} 
Schlussfolgerung: Eine individuelle, der klinischen Situation angepasste Ernährungstherapie ist ein wesentlicher Baustein in der multimodalen Therapie der akuten und chronischen Pankreatitis.

\section{Methodik}

$\nabla$

Die vorliegende Leitlinie ist eine Weiterentwicklung der DGEMLeitlinien enterale [1] und parenterale Ernährung [2] in der Gastroenterologie sowie der ESPEN-Leitlinien zur enteralen [3] und parenteralen [4] Ernährung bei Pankreatitis. Es handelt sich hierbei um eine S3-Leitlinie der DGEM (AWMF-Registernummer 073/025). Die Methodik ist im Leitlinienreport ausführlich beschrieben, wo sich auch die Suchstrategien und Evidenztabellen finden. Der Leitlinienreport ist über die Internetseite der Arbeitsgemeinschaft der Wissenschaftlichen Medizinischen Fachgesellschaften e.V. (AWMF) abrufbar (www.awmf.org, AWMF-Registernummer der Fachgesellschaft 073). Ein Auszug zum methodischen Vorgehen bei der Leitlinienerstellung wurde bereits in der Aktuellen Ernährungsmedizin veröffentlicht [5].

Neben dem Empfehlungsgrad wird auch die Outcome-Bewertung bei den Empfehlungen mit angegeben (Biomedizinische Endpunkte [BM], Patientenzentriertes Outcome [PC], Gesundheitsökonomische Parameter [HE], Medizinische Entscheidungsfindung [DM], Mehr-Komponenten-Outcome-Modelle [MC]) [6].

\section{Akute Pankreatitis}

2.1 Welche Methode ist am besten geeignet, den Ernährungszustand bei Patienten mit akuter Pankreatitis zu objektivieren?

\section{Empfehlung 1:}

Zur Evaluation des ernährungsmedizinischen Risikos bzw. des vorliegenden Ernährungszustands von Patienten mit akuter Pankreatitis können die allgemeinen in der klinischen Praxis etablierten Methoden, wie z. B. Nutritional Risk Screening (NRS-2002) oder Subjective Global Assessment (SGA), zusammen mit dem Verlauf des Body-Mass-Indexes (BMI) eingesetzt werden. [C (BM, QL, HE); starker Konsens]

\section{Empfehlung 2:}

Bei klinisch kritischem Verlauf kann im Einzelfall auch eine Bioelektrische Impedanzanalyse (BIA-Messung) eingesetzt werden. [C (BM, QL, HE); starker Konsens]

Kommentar: Da es in der vorliegenden Literatur keine systematischen Untersuchungen zum Einfluss des Ernährungszustands auf den klinischen Verlauf und die Prognose von Patienten mit akuter Pankreatitis gibt, können hierzu keine spezifischen Angaben gemacht werden. In Analogie zu Untersuchungen bei anderen schwerwiegenden akuten Erkrankungen ist es wahrscheinlich, dass bei Patienten mit akuter nekrotisierender Pankreatitis das Vorliegen einer relevanten Unter-/Mangelernährung den klinischen Verlauf und die Prognose der Erkrankung negativ beeinflusst.

Patienten mit leichter akuter Pankreatitis haben in der Regel keinen relevant erhöhten Nährstoff- und Energiebedarf und der in- dividuelle Ernährungszustand wird mit Ausnahme protrahierter Verlaufsformen nicht relevant beeinflusst [3] (II).

Bei Patienten mit schwerer nekrotisierender Pankreatitis führen inflammatorischer Stress und Schmerz zu einer Erhöhung der basalen Stoffwechselaktivität mit entsprechend gesteigertem Energieumsatz und Proteinkatabolismus [7, 8] (II). Je nach Schwere und Verlauf der Erkrankung kann es zu einer progredienten Verschlechterung des Ernährungszustands betroffener Patienten kommen.

Zur Bestimmung des individuellen Ernährungszustands bei Patienten mit akuter Pankreatitis wird auf die allgemeinen in der klinischen Praxis etablierten Methoden verwiesen [9].

\subsection{Wann ist eine Ernährungstherapie indiziert bzw. kontraindiziert?}

\section{Empfehlung 3:}

Patienten mit milder akuter Pankreatitis benötigen keine gezielte Ernährungsintervention und sollen unabhängig vom Verlauf der Lipase- und/oder Amylaseaktivität frühzeitig eine leichte Vollkost bekommen; eine Ernährung über enterale Sondensysteme oder über parenterale Zugänge ist nicht indiziert.

[A (BM); starker Konsens]

Kommentar: Die meisten Patienten präsentieren sich mit einer leichten oder moderaten akuten Pankreatitis, die innerhalb von 5-7 Tagen mit einer Restitutio ad integrum ausheilt. Hier wurde früher ein eher zurückhaltendes Vorgehen bei der Wiederaufnahme der oralen Kostzufuhr unter der Vorstellung der Vermeidung der Stimulation der Pankreassekretion empfohlen. Prospektive Daten, die dieses Vorgehen stützten, lagen allerdings nicht vor.

Aktuelle Studien haben jetzt ein vereinfachtes und patientenzentriertes Vorgehen bei einem Kostaufbau nach Pankreatitis untersucht $[10,11]$. In einer deutschen offenen randomisierten Multicenterstudie konnte gezeigt werden, dass bei milder akuter Pankreatitis der Wunsch des Patienten nach Wiederaufnahme der oralen Ernährung eine wichtigere Rolle spielen kann als bisher und dass dieses unabhängig von der Normalisierung der Serumlipase erfolgen kann [12]. Des Weiteren zeigt eine Studie mit 101 Patienten, dass weiche Nahrung im Vergleich zu einer flüssigen Nahrung von Vorteil ist [13]. Der Kostaufbau kann demnach mit einer leichten Vollkost erfolgen.

\section{Empfehlung 4:}

Bei Patienten mit schwerer nekrotisierender Pankreatitis soll frühzeitig (innerhalb von 24-48 Stunden) eine enterale Ernährungsstrategie begonnen werden.

[A (BM); starker Konsens]

\section{Empfehlung 5:}

Der Stellenwert und der Zeitpunkt einer dualen (enteralen und parenteralen) Ernährung sind nicht klar. Der Beginn einer kombinierten Ernährung sollte nach 3 Tagen erwogen werden und spätestens nach einer Woche hypokalorischer Ernährung begonnen werden.

[KKP (BM); starker Konsens] 


\section{Empfehlung 6:}

Eine totale parenterale Ernährung soll nur in dem sehr seltenen Fall einer klinisch nicht durchführbaren minimalen enteralen $\mathrm{Er}$ nährung erfolgen.

[A (BM); Konsens]

Kommentar: Eine Vielzahl von prospektiven klinischen Studien und mittlerweile auch mehrere systematische Metaanalysen u.a. auch nach Cochrane-Standard [14-17] belegen überzeugend, dass eine innerhalb von 24-48 Stunden begonnene enterale Ernährungstherapie mit nährstoffdefinierter, hochmolekularer Sondennahrung signifikant die Infektionsrate sowie signifikant die Mortalität von Patienten mit akuter nekrotisierender Pankreatitis senkt. Die aktuellste Metaanalyse von Al-Omran et al. [17] nach Cochrane-Standard hat 8 randomisierte kontrollierte klinische Studien mit 348 Patienten analysiert und fand bei frühzeitiger enteraler Ernährung im Vergleich zur bisher durchgeführten totalen parenteralen Ernährung eine Senkung der Mortalität um 50\% (OR 0,50 [95\%-KI: 0,28-0,91]). Wenn nur Studien mit Patienten mit schwerer akuter nekrotisierender Pankreatitis eingeschlossen werden, wird die Odds Ratio (OR) sogar auf 0,18 (95\%-KI: 0,006-0,58) signifikant gesenkt. Neben der signifikanten Senkung der Mortalität findet sich auch eine signifikante Senkung der Infektionsrate (OR 0,39 [95\%-KI: 0,23-0,65]), des Multiorganversagens (OR 0,55 [95\%-KI: 0,37-0,81]) sowie der Operationsnotwendigkeit (OR 0,44 [95\%-KI: 0,29-0,67]). Unter kritischer Würdigung der jetzt vorliegenden randomisierten kontrollierten Studien zur Frage der klinischen Effizienz einer frühzeitigen enteralen Ernährungsstrategie im Vergleich zur bisher durchgeführten konventionell totalen parenteralen Ernährung sowie der aktuell verfügbaren großen Metaanalysen ist wissenschaftlich überzeugend belegt, dass die frühzeitige enterale Ernährung bei Patienten mit akuter nekrotisierender Pankreatitis signifikant die harten klinischen Outcomeparameter Infektionsrate, Operationsnotwendigkeit, Multiorganversagen und vor allen Dingen auch Mortalität beeinflusst. Li et al. [18] konnten in einer Metaanalyse von 11 Studien mit insgesamt 775 Patienten zeigen, dass eine frühzeitige, innerhalb der ersten 48 Stunden durchgeführte enterale Ernährung die Endpunkte Morbidität und Mortalität im Vergleich zu Patienten, die innerhalb von 72 Stunden enteral ernährt werden, deutlich senkt. Diese Studie bestätigt, dass bei klinisch stabilen Patienten so früh wie möglich mit der enteralen Ernährung begonnen werden sollte. Damit ist unter klinischen Gesichtspunkten die frühzeitige enterale Ernährung bei Patienten mit akuter nekrotisierender Pankreatitis hocheffektiver, integraler Bestandteil der medizinisch notwendigen Therapiestrategie.

Es sind keine Kontraindikationen für den Beginn einer minimalen enteralen Ernährung bei Patienten mit akuter Pankreatitis bekannt, die über die allgemeingültigen Kontraindikationen zur enteralen Ernährung hinausgehen [15, 16] (II).

In Abhängigkeit von der Schwere der Erkrankung weisen Patienten mit akuter Pankreatitis sehr häufig Subileus-/Ileussymptome auf. Das Vorliegen einer Ileussymptomatik im Rahmen einer akuten Pankreatitis ist per se keine Kontraindikation für die Durchführung einer minimalen enteralen Ernährung. Im Gegenteil: Wie bei anderen schweren Erkrankungen sowie in der postoperativen Betreuung fördert eine frühzeitige enterale Ernährung auch bei Patienten mit akuter Pankreatitis den Erhalt der gastrointestinalen Integrität und damit die Barrierefunktion, steigert die intestinale Motilität und hat in mehreren Studien und
Metaanalysen nachgewiesenermaßen einen relevanten Einfluss auf klinisch wichtige Outcomeparameter wie Komplikationsrate, Morbidität und Mortalität [14-17].

Dezidierte Studien zur Beeinflussung des Ernährungszustandes unter einer enteralen Ernährung bei Patienten mit akuter Pankreatitis liegen nicht vor. Aufgrund indirekter Daten der parenteralen Ernährung und der Verbesserung der Outcomeparameter kann jedoch von einem positiven Effekt auf den Ernährungszustand ausgegangen werden.

Nach unseren heutigen wissenschaftlichen Vorstellungen besteht der klinische Nutzen einer frühzeitigen enteralen Ernährung primär darin, den Magen-Darm-Trakt strukturell und funktionell in Takt zu halten, um seine Integrität als klinisch wichtige Grenzfläche, insbesondere in Bezug auf eine Translokation von Darmkeimen, zu erhalten. Unter dieser Vorstellung ist die frühzeitige enterale Ernährung primär Teil einer präventiven Behandlung und weniger Teil einer bloßen Ernährungsstrategie. Aufgrund der klinischen Erfahrung sowie der Daten aus Studien mit anderen intensivmedizinischen Krankheitsbildern kann für die klinische Praxis davon ausgegangen werden, dass eine im Rahmen des Postaggressionsstoffwechsels adäquate enterale oder kombinierte enteral-parenterale Ernährung den Ernährungszustand der betroffenen Patienten positiv beeinflusst.

Im Rahmen der akuten Pankreatitis ist eine Besserung der Pankreasfunktion durch eine enterale Ernährung nicht möglich. Gleichwohl scheint eine enterale Ernährung die Pankreasfunktion nicht negativ zu beeinflussen, da eine etwaige Stimulation der Pankreassekretion im Rahmen einer akuten Pankreatitis keine klinisch erkennbare Relevanz zeigt.

\subsection{Wie soll die enterale Ernährung durchgeführt werden?}

\section{Empfehlung 7:}

Für eine enterale Ernährung sollen hochmolekulare (nährstoffdefinierte) Diäten verwendet werden [A (BM); starker Konsens]; nur bei Unverträglichkeit kann auf niedermolekulare (chemisch definierte) Diäten ausgewichen werden [KKP; starker Konsens].

\section{Empfehlung 8:}

Die Datenlage bezüglich Immunonutrition und Prä-, Pro- und Synbiotika ist aktuell kontrovers. Die Gabe kann daher derzeit nicht empfohlen werden.

[KKP; starker Konsens]

Kommentar: Es liegen mehrere prospektive randomisierte Studien vor zur Frage, welche Sondennahrungen bei akuter Pankreatitis eingesetzt werden sollen. Jedoch nur eine Studie mit relativ geringer Fallzahl $(n=30)$ vergleicht direkt eine hochmolekulare (nährstoffdefinierte) Sondennahrung mit einer niedermolekularen (chemisch definierten) Sondennahrung zur enteralen Ernährung bei milder/moderater akuter Pankreatitis [19]. Obwohl diese Studie einen leichten Vorteil für eine niedermolekulare Sondennahrung zeigt, kommt eine indirekte adjustierte Metaanalyse mit 428 Patienten zu dem Schluss, dass kein Unterschied bzgl. Toleranz, Infektionsraten und Letalität zwischen nieder- oder hochmolekularer Sondennahrung besteht [20].

Die vorliegenden Studien zur Gabe von Pro- und Präbiotika bei Patienten mit schwerer akuter Pankreatitis zusammengefasst in einer Metaanalyse [21] konnten keinen klinischen Vorteil in Be- 
zug auf infektiöse und nicht infektiöse Komplikationen, Mortalität oder die Krankenhausaufenthaltsdauer belegen. Einige Studien weisen sogar signifikante negative Effekte durch Prä-/Probiotika auf [22]. Ebenso ist der klinische Nutzen einer Immunonutrition zusammengefasst in einer Metaanalyse [23] nicht belegt. Auch hier gibt es Studienergebnisse mit einem Trend zu eher negativen Effekten, z.B. durch gezielte Substitution mit Selen, NAzetylzysteinen und Vitamin C [24].

\section{Empfehlung 9:}

Eine enterale Sondenernährung soll primär gastral erfolgen. [A (BM); starker Konsens]

\section{Empfehlung 10:}

Bei gastraler Intoleranz soll die Ernährung über ein nasojejunales Sondensystem bevorzugt werden.

[A (BM); starker Konsens]

Kommentar: In mehreren prospektiven klinischen Studien sowie mittlerweile in 2 Metaanalysen [25, 26] konnte überzeugend belegt werden, dass eine nasogastrale Ernährung ebenso gut verträglich, sicher und effektiv ist wie eine nasojejunale Ernährung bei Patienten mit schwerer akuter Pankreatitis. Aufgrund dieser Daten kann primär mit einer gastralen Ernährung bei Patienten mit schwerer akuter Pankreatitis begonnen und diese bei guter Verträglichkeit fortgeführt werden. Sollte es zum Auftreten einer gastralen Intoleranz kommen, soll auf eine Ernährung über ein nasojejunales Sondensystem gewechselt werden.

2.4 Wie soll die orale Ernährung nach enteraler Sondenernährung (inkl. Kostaufbau und ggf. Fermentsubstitution) durchgeführt werden?

\section{Empfehlung 11:}

Ein spezifischer Kostaufbau sowie eine passagere Fermentsubstitution sollte in der Regel nicht durchgeführt werden. [B (BM); starker Konsens]

\section{Empfehlung 12:}

Nach Aufbau der enteralen Sondenernährung kann in Abhängigkeit vom klinischen Verlauf auf eine leichte orale Vollkost umgestellt werden.

[C (BM); starker Konsens]

Belege für die klinische Notwendigkeit eines spezifischen Kostaufbaus im Übergang zur oralen Ernährung liegen nicht vor. Bei Hinweisen auf eine klinisch relevante Maldigestion im Verlauf einer schweren akuten Pankreatitis kann eine Fermentsubstitution erfolgen.

[C (BM); starker Konsens]

Kommentar: Spezifische Daten oder Untersuchungen zum Kostaufbau bzw. Umstellung von einer enteralen Ernährung auf eine orale Kost liegen nicht vor. Die vorliegende Empfehlung basiert auf dem Analogschluss unter Berücksichtigung der Datenlage beim Kostaufbau bei leichter und moderater Pankreatitis (siehe 3.2; Empfehlung 3). Ein weiteres Argument für die Empfehlung für einen vereinfachten Kostaufbau liegt darin, dass die enterale Sondenernährung in der Regel mit einer hochmolekularen Son- dennahrung gastral durchgeführt wird, was in der Zusammensetzung einer normalen Ernährung entspricht.

\subsection{Wie soll die parenterale Ernährung durchgeführt werden?}

\section{Empfehlung 13:}

Die Art und Weise der parenteralen Ernährung bei akuter Pankreatitis soll nach den allgemeinen Regeln der parenteralen Ernährung erfolgen.

[KKP; starker Konsens]

\section{Empfehlung 14:}

Die zugeführte Energiemenge sollte der metabolischen Situation angepasst werden. Der Flüssigkeitsbedarf sollte individuell an die klinische Situation angepasst werden.

[KKP; starker Konsens]

\section{Empfehlung 15:}

Glukose soll aufgrund der sofortigen Verfügbarkeit für die Energiegewinnung als ein wesentlicher Makronährstoff für die parenterale Ernährung bei akuter Pankreatitis verwendet werden und stimuliert die Pankreassekretion nicht. Die Zufuhrrate soll bei

$2-4 \mathrm{~g}$ pro kg Körpergewicht liegen.

[A; starker Konsens]

\section{Empfehlung 16:}

Zur Abwendung eines Proteinkatabolismus soll in Abhängigkeit vom Ernährungszustand die Zufuhr von 1,2-1,5g Protein pro kg Körpergewicht erfolgen.

[A; starker Konsens]

\section{Empfehlung 17:}

Die intravenöse Gabe von Fetten soll erfolgen, da sie essenziell zur Deckung des Energiebedarfs und der Zufuhr essenzieller Fettsäuren ist.

[KKP; starker Konsens]

\section{Empfehlung 18:}

Die Infusionsraten von Lipiden sollten zwischen 0,8 und $1,5 \mathrm{~g}$ pro kg Körpergewicht liegen und nur unter strengem Monitoring der Plasmatriglyzeride erfolgen. Plasmatriglyzeridspiegel über 12 $\mathrm{mmol} / \mathrm{L}$ (unter laufender Infusion) sollten vermieden werden und können zum Pausieren der Lipidinfusion Anlass geben.

[C; starker Konsen]

Kommentar: Die Grundprinzipien der parenteralen Ernährung bei einer akuten Pankreatitis entsprechen dem Vorgehen bei kritisch kranken Patienten, daher wird hier auf die DGEM-Leitlinie „Klinische Ernährung in der Intensivmedizin“ verwiesen.

Der Gesamtenergieumsatz im Rahmen einer akuten Pankreatitis entspricht in der Regel etwa $25-30 \mathrm{kcal} / \mathrm{kg}$ KG/Tag [7]. Die parenterale Zufuhr von Lipidemulsionen hat nach der derzeitigen Studienlage keinen negativen Effekt auf die Pankreasfunktion und den Verlauf der Erkrankung. Durch eine parenterale Ernährung kann der Ernährungszustand des Patienten erhalten oder 
verbessert werden $[4,27]$ (IIb). Eine Stimulation der exokrinen Pankreasfunktion unter parenteraler Ernährung ist aufgrund der derzeitigen Daten nicht zu erwarten.

Patienten mit akuter Pankreatitis, die längere Zeit ausschließlich parenteral ernährt werden, profitieren von einer zusätzlichen intravenösen Gabe von Glutamin in einer Dosis von $0,2-0,5 \mathrm{~g} / \mathrm{kg}$ KG/Tag. Die Gabe von Glutamin in dieser Patientengruppe ist mit einer Reduktion von infektiösen Komplikationen und einer reduzierten Mortalität assoziiert [28].

\section{Empfehlung 19:}

Die Supplementierung von Mikronährstoffen sowie Mineralien und Spurenelementen in Höhe der allgemein empfohlenen Zufuhrrate kann in jedem Fall täglich erfolgen.

[C; starker Konsens]

Eine hoch dosierte generelle Supplementierung, besonders von antioxidativ wirkenden Vitaminen, sollte nicht erfolgen. [B; starker Konsens]

Kommentar: In 2 prospektiven randomisierten klinischen Studien war kein erkennbarer Vorteil einer Substitution von antioxidativen Substanzen in pharmakologischen Dosen (z. B. Vitamin C 1000 - 2000 mg/Tag; Vitamin E 200 mg/Tag, Selen 200-1000 $\mu$ g/ Tag) zu erkennen $[24,29]$.

Während bei einer enteralen Ernährung von $1500 \mathrm{~mL}$ oder mehr in der Regel die Zufuhr der empfohlenen Tagesdosis von Vitaminen und Spurenelementen gedeckt ist, ist es notwendig bei einer geringeren enteralen Ernährung mit oder ohne parenteraler Ernährung die Tagesdosis von Vitaminen und Spurenelementen entsprechend zu substituieren.

\subsection{Monitoring}

\section{Empfehlung 20:}

Auf ein spezifisches Monitoring der enteralen sowie parenteralen Ernährungstherapie bei Patienten mit akuter Pankreatitis im Vergleich zu anderen Intensivpatienten kann verzichtet werden. [C; starker Konsens]

Davon ausgeschlossen ist das Monitoring der Volumensubstitution. Dieses sollte bei schweren Verläufen mit besonderer Sorgfalt erfolgen.

[B; starker Konsens]

\section{Chronische Pankreatitis}

3.1 Welche Methode ist am besten geeignet, den Ernährungszustand bei Patienten mit chronischer Pankreatitis zu objektivieren?

\section{Empfehlung 21:}

Der Ernährungszustand bei chronischer Pankreatitis kann mit anthropometrischen, biochemischen, klinischen und diätetischen Parametern evaluiert werden.

[KKP; starker Konsens]

Kommentar: Das Ausmaß von Mangelernährung bei chronischer Pankreatitis korreliert mit der Komplikationsrate und ist mit einer erhöhten Mortalität assoziiert [30] (IIb). Daten aus einer deutschen Rehabilitationsklinik (74\% alkoholische chronische Pankreatitis) zeigen bei $32 \%$ der Patienten einen $\mathrm{BMI}<20 \mathrm{~kg} / \mathrm{m}^{2}$, 57\% hatten Diarrhö und 24\% eine schwere Steatorrhö (fäkaler Fettverlust $>30 \mathrm{~g}$ Fett/Tag) [31]. Ebenfalls ein hoher Anteil an unterernährten Patienten $\left(23 \% \mathrm{BMI}<20 \mathrm{~kg} / \mathrm{m}^{2}\right)$ fand sich in einer Gruppe von Patienten mit chronischer Pankreatitis, die zu einer Operation (Pankreaskopfresektion) anstanden. Auch nach der Operation fand sich nur eine mediane Gewichtszunahme von $2 \mathrm{~kg}[32]$.

Untersuchungen zur Körperzusammensetzung bei Patienten mit chronischer Pankreatitis dokumentieren eine Reduktion der fettfreien Masse sowie der Fettmasse und erniedrigte Serumspiegel für fettlösliche Vitamine in Abhängigkeit vom Ausmaß der exokrinen Insuffizienz [33].

Gewichtsverlust ist mit einer Reduktion der funktionellen Reserve und der Lebensqualität assoziiert [34]. 34\% der Patienten zeigten relevanten Gewichtsverlust und $46 \%$ berichteten über Fatigue und körperliche Schwäche. Durch den Mangel an Mikronährstoffen und zusätzlich durch die chronische Entzündung ist das Risiko einer Osteoporose und Osteopenie erhöht [33].

Vergleichende spezielle Untersuchungen, welche Methode zur besten Charakterisierung des Ernährungszustands geeignet ist, liegen nicht vor, sodass hier allgemeine Empfehlungen [9] gelten.

\subsection{Wann ist eine Ernährungstherapie indiziert bzw. kontraindiziert?}

\section{Empfehlung 22:}

Sollte es bei Patienten mit Malnutrition trotz individualisierter Ernährungsberatung und adäquater Pankreasenzymsubstitutionstherapie zu keiner Besserung der Nahrungszufuhr und der Nährstoffabsorption kommen, kann der Beginn einer enteralen Ernährungstherapie mit einer hochmolekularen oder auch niedermolekularen Sondennahrung erwogen werden.

[KKP; starker Konsens]

\section{Empfehlung 23:}

Eine parenterale Ernährungstherapie kann bei Kontraindikation für eine enterale Ernährung oder unzureichender enteraler Ernährungstherapie begonnen werden.

[C (BM); starker Konsens]

\section{Empfehlung 24:}

Die Kontraindikationen für eine enterale und parenterale Ernährungstherapie bei chronischer Pankreatitis entsprechen den allgemeinen Kontraindikationen für enterale und parenterale Ernährung.

[KKP; starker Konsens]

Kommentar: Die progressive exokrine und endokrine Pankreasdysfunktion bei chronischer Pankreatitis beeinträchtigt nachhaltig Digestion und Absorption von Nährstoffen. Patienten in der terminalen Phase der chronischen Pankreatitis weisen häufig eine Eiweißmangelernährung auf. Bis zu 50\% der Patienten mit chronischer Pankreatitis haben einen erhöhten Ruhe-Energieumsatz. Darüber hinaus besteht bei schwerer exokriner Pankreasinsuffizienz häufig ein Vitamin-A-, -D-, -E- und -K-Mangel sowie einen Mangel an Kalzium, Magnesium, Zink, Thiamin und Folsäure [30] (III). 
Eine orale Nahrungssupplementation aber auch eine individuelle Ernährungsberatung kann den Ernährungszustand verbessern, die Fettmaldigestion reduzieren und die Schmerzen positiv beeinflussen [35-37] (Ib). Wenn eine orale Nahrungszufuhr nicht möglich ist, scheint eine nasojejunale enterale Ernährung den Ernährungszustand und die Schmerzen zu verbessern [35 - 37] (III). Neben einer Enzymsupplementation beinhaltet die Therapie der chronischen Pankreatitis eine individuelle Ernährungsberatung. Wesentliche Eckpunkte der Beratung sind eine ausreichende Kalorienzufuhr mit $25-30 \mathrm{kcal} / \mathrm{kg} \mathrm{KG} / \mathrm{Tag}$ (ggf. bis $35 \mathrm{kcal} / \mathrm{kg} \mathrm{KG} /$ Tag) und eine Eiweißzufuhr von 1,5 g/ kg KG/Tag. Hierbei ist auf eine adäquate Fettzufuhr im Rahmen der individuellen Verträglichkeit zu achten, ggf. kann die Kohlenhydratzufuhr angepasst werden. Wenn diese Ziele nicht über diätetische Maßnahmen erreicht werden können, besteht die Möglichkeit des Einsatzes von Trinknahrung (orale bilanzierte Diäten) [35].

In prospektiven unkontrollierten Untersuchungen [36, 37] konnte eine signifikante Gewichtszunahme und Abnahme der Schmerzsymptomatik unter einer längerfristigen enteralen Sondenernährung bei Patienten mit chronischer Pankreatitis und unzureichender oraler Nahrungsaufnahme gezeigt werden.

\subsection{Wie soll die orale Ernährung (inkl. Fermentsubstitu- tion) durchgeführt werden?}

\section{Empfehlung 25:}

Eine prinzipielle Fettrestriktion sollte nicht erfolgen, wenn die exokrine Pankreasinsuffizienz durch Enzymgabe ausreichend kompensiert ist. Eine Alkoholabstinenz sowie die Einnahme kleiner häufiger Mahlzeiten sollte erfolgen.

[B (BM, QL); starker Konsens]

Siehe hierzu die Leitlinie der DGVS zum Thema chronische Pankreatitis [38].

\subsubsection{Enzymsubstitution}

\section{Empfehlung 26:}

Pankreasenzyme sollen supplementiert werden bei Patienten, bei denen eine deutliche Steatorrhö besteht bzw. anzunehmen ist (Nachweisverfahren: Stuhlfette >15 g/Tag, sofern verfügbar; sonst: pathologische qualitative Stuhlfettausscheidung oder pathologischer Pankreasfunktionstest in Kombination mit klinischen Zeichen der Malabsorption) [38].

[A (BM, QL); starker Konsens]

\section{Empfehlung 27:}

Auch bei geringerer pathologischer Stuhlfettausscheidung (7 $15 \mathrm{~g} / \mathrm{Tag}$ ) soll Pankreatin supplementiert werden, wenn Zeichen der Malabsorption bestehen (z. B. Gewichtsverlust) oder der Patient abdominelle Symptome hat, die auf die Maldigestion und Malabsorption zurückgeführt werden können [38]. [A (BM, QL); starker Konsens]

Kommentar: Eine Reduktion der Nahrungsfette erhöht das Risiko einer hypokalorischen Ernährung und sollte daher vermieden werden. Bei Patienten mit chronischer exokriner Pankreasinsuffizienz, rezentem Gewichtsverlust, einer erhöhten fäkalen Fettexkretion $(>7 \mathrm{~g} / \mathrm{Tag})$ unter einer Diät mit maximal $100 \mathrm{~g}$ Fett pro

\section{Empfehlung 28:}

Der Enzymgehalt von Pankreasenzympräparaten wird anhand der Lipaseaktivitat gemessen. Pro Hauptmahlzeiten sollten mindestens 20000 - 40000 Einheiten (Ph. Eur.) verabreicht werden, für die Verdauung kleinerer Zwischenmahlzeiten mindestens 10000 (bis 20000$)$ Lipaseeinheiten [38].

[B (BM, QL); Konsens]

Tag und relevanten Steatorrhö-bedingten klinischen Symptomen sollte eine Pankreasenzymsubstitutionstherapie begonnen werden $[38,39]$. Eine frühzeitige Pankreasenzymsubstitutionstherapie bei asymptomatischer Steatorrhö und laborchemischen Zeichen einer Resorptionsstörung (z.B. erniedrigte fettlösliche Vitaminspiegel) kann zu einer Normalisierung dieser Laborparameter führen [40]. Die Pankreasenzymsubstitutionstherapie sollte vorwiegend in Form der modernen magensaftresistenten Mikropellets und zeitgleich während jeder Nahrungsaufnahme verabreicht werden [41] [IV].

Siehe hierzu auch die Leitlinie der DGVS zum Thema chronische Pankreatitis [38].

\subsection{Wie soll die enterale Ernährung durchgeführt werden?}

\section{Empfehlung 29:}

Die enterale Ernährungstherapie sollte primär mit einer Standardsondennahrung und bei Verdacht auf Magenentleerungsstörung initial ggf. über eine nasojejunale Sonde durchgeführt werden.

[B (BM); starker Konsens]

Das Ziel der Kalorienzufuhr sollte 25 - $30 \mathrm{kcal} / \mathrm{kg} \mathrm{KG/Tag} \mathrm{betragen.}$ [KKP; starker Konsens]

Bei Unverträglichkeit der hochmolekularen Standardsondennahrung kann eine fettreduzierte bzw. niedermolekulare nasojejunale enterale Ernährung eingesetzt werden.

[C; starker Konsens]

\section{Empfehlung 30:}

Eine enterale Langzeiternährung kann über eine perkutane Gastrostomie (PEG-Sonde) mit einer jejunalen Zusatzsonde oder über eine direkte perkutane Jejunostomie durchgeführt werden. [C (BM, QL); starker Konsens]

Kommentar: In prospektiven unkontrollierten Untersuchungen $[36,37]$ bei Patienten mit chronischer Pankreatitis und unzureichender oraler Nahrungsaufnahme findet sich kein Hinweis auf eine höhere Komplikationsrate bei Anlage einer perkutanen endoskopischen Gastrostomie oder Jejunostomie.

Vergleichende Untersuchungen zur Art der Sondennahrung liegen nicht vor. Im Analogschluss zur akuten Pankreatitis erscheint ein primärer Versuch mit hochmolekularer Sondennahrung gerechtfertigt (vergleiche hierzu 3.3; Empfehlung 8). 


\subsection{Wie soll die parenterale Ernährung durchgeführt werden?}

\section{Empfehlung 31:}

Die parenterale Ernährung kann gemäß den allgemeinen Standards durchgeführt werden.

[C; starker Konsens]

Kommentar: Eine parenterale Ernährung kann den Ernährungszustand verbessern und mangelernährungsbedingte Komplikationen verhindern [30] (III).

Die Indikation zu einer parenteralen Ernährung bei Patienten mit chronischer Pankreatitis ergibt sich ausschließlich über eine längerfristig (>6 Tage) nicht ausreichende Möglichkeit zur oralen oder enteralen Ernährung. Kontrollierte Studien liegen hierzu nicht vor.

\subsection{Monitoring}

\section{Empfehlung 32:}

Das Monitoring einer enteralen oder parenteralen Ernährung kann analog dem standardgemäßen Vorgehen erfolgen (siehe hierzu [42]). Es ist allerdings besonders der Glukosestoffwechsel aufgrund des Risikos eines pankreopriven Diabetes regelmäßig zu überprüfen und bei enteraler Ernährung zusätzlich auf Zeichen einer Fettmalabsorption (Diarrhö, Fettstühle, Meteorismus) zu achten.

[C (BM, QL); starker Konsens]

Kommentar: Im Rahmen einer chronischen Pankreatitis kommt es häufiger zu Störungen des Glukosestoffwechsels [43]. Neben dem Bild des klassischen pankreopriven Diabetes mit konsekutiver Insulintherapie besteht auch ein erhöhtes Risiko für Hypoglykämien aufgrund mangelnder Glukagonfreisetzung.

\section{Interessenkonflikte}

Gemäß den AWMF-Richtlinien wurden die bestehenden potenziellen Interessenkonflikte zu Beginn der Leitlinienarbeit von allen Autoren dargelegt. Die Autoren haben bei folgenden Punkten entsprechende Angaben gemacht:

Berater- bzw. Gutachtertätigkeit oder Mitglied eines wissenschaftlichen Beirats eines Unternehmens: J. Ockenga, S. C. Bischoff, H. Lochs.

Vortragshonorare von Unternehmen: J. Ockenga, C. Löser, M. Kraft, C. Madl, S. C. Bischoff, H. Lochs, A. Weimann.

Finanzielle Zuwendungen für Forschungsvorhaben vonseiten eines Unternehmens: C. Löser, M. Kraft, S. C. Bischoff, A. Weimann.

Einzelheiten sind im Leitlinienreport des Leitlinien-Updates Klinische Ernährung, der über die Internetseite der AWMF abrufbar ist, hinterlegt.

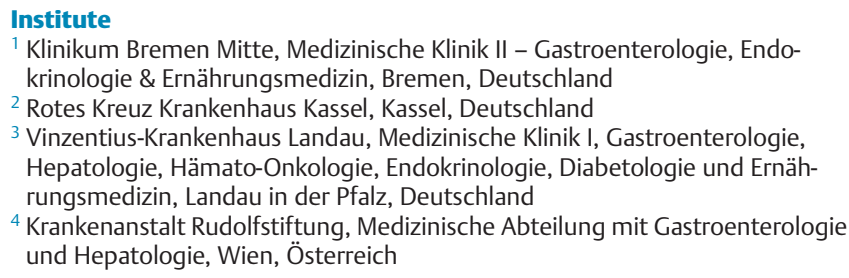


Evidenztabelle 1 Ernährung bei milder akuter Pankreatitis.

\begin{tabular}{|c|c|c|c|c|c|c|}
\hline Referenz & Evidenzgrad & Studientyp & Teilnehmer & Intervention & Zielgröße & Hauptergebnis \\
\hline $\begin{array}{l}\text { Jacobson et al. } \\
2007[10]\end{array}$ & $\mathrm{Ib}$ & $\begin{array}{l}\text { randomisiert, } \\
\text { kontrolliert }\end{array}$ & $\begin{array}{l}121 \text { Patienten mit milder } \\
\text { akuter Pankreatitis } \\
\text { KG: } n=66 \\
\text { IG: } n=55\end{array}$ & $\begin{array}{l}\text { IG: fettreduzierte Kost: } \\
1200 \text { Kalorien, } 35 \mathrm{~g} \text { Fett/ } \\
\text { Tag; } \\
\text { KG: klare Flüssigkeiten: } \\
588 \text { Kalorien, } 2 \text { g Fett/ } \\
\text { Tag }\end{array}$ & $\begin{array}{l}\text { wiederkehrende Schmerzen, Notwendigkeit } \\
\text { die Nahrungsaufnahme zu beenden, Kran- } \\
\text { kenhausverweildauer nach Wiederaufnah- } \\
\text { me der Nahrungszufuhr, Notwendigkeit der } \\
\text { Wiedereinlieferung innerhalb von } 28 \text { Tagen } \\
\text { nach Wiederaufnahme der Nahrungszufuhr }\end{array}$ & $\begin{array}{l}\text { Beendigung der Ernährung aufgrund von Schmerzen } \\
\text { oder Übelkeit war in beiden Gruppen ähnlich ( } 6 \% \text { bei KG, } \\
11 \% \text { bei IG; } p=0,51 \text { ). } \\
\text { Durchschnittliche Krankenhausverweildauer nach Wie- } \\
\text { deraufnahme der Nahrungszufuhr war in beiden Grup- } \\
\text { pen identisch ( } 1 \text { Tag, IQR } 1-2 ; p=0,77 \text { ). } \\
\text { Patienten der IG nahmen während ihrem ersten Essen } \\
\text { und des ersten Studientags bedeutend mehr Kalorien } \\
\text { und Fett auf als Patienten der KG-Gruppe. } \\
\text { Keine Unterschiede zwischen den Gruppen bei der Wie- } \\
\text { dereinlieferung innerhalb von } 28 \text { Tagen nach Wieder- } \\
\text { aufnahme der Nahrungszufuhr. }\end{array}$ \\
\hline $\begin{array}{l}\text { Moraes et al. } 2010 \\
\text { [11] }\end{array}$ & $\mathrm{Ib}$ & $\begin{array}{l}\text { randomisiert, } \\
\text { kontrolliert }\end{array}$ & $\begin{array}{l}210 \text { Patienten mit milder } \\
\text { akuter Pankreatitis, pro } \\
\text { Arm: } n=70\end{array}$ & $\begin{array}{l}\text { IG: feste Nahrung; } \\
\text { KG1: klare Flüssigkeiten; } \\
\text { KG2: weiche Nahrung }\end{array}$ & $\begin{array}{l}\text { Schmerzen, Nahrungsaufnahme, Kranken- } \\
\text { hausverweildauer, Wiederauftreten von } \\
\text { Schmerzen } 7 \text { Tage nach Entlassung }\end{array}$ & $\begin{array}{l}\text { Keine Unterschiede zwischen den } 3 \text { Diäten hinsichtlich } \\
\text { Wiederauftretens von Schmerzen nach Nahrungsauf- } \\
\text { nahme. } \\
\text { Personen der IG nahmen an Studientag } 1 \text { und } 2 \text { signifi- } \\
\text { kant mehr Kalorien und Fett auf ( } p<0,001 \text { ). } \\
\text { Eine kürzere Krankenhausverweildauer wurde bei Pa- } \\
\text { tienten der IG ohne Wiederauftreten von abdominalen } \\
\text { Schmerzen beobachtet (Median - } 1,5 \text { Tage; } p=0,000 \text { ). }\end{array}$ \\
\hline $\begin{array}{l}\text { Teich et al. } 2010 \\
\text { [12] }\end{array}$ & $\mathrm{Ib}$ & $\begin{array}{l}\text { randomisiert, } \\
\text { kontrolliert }\end{array}$ & $\begin{array}{l}143 \text { Patienten mit milder } \\
\text { akuter Pankreatitis } \\
\text { KG: } \mathrm{n}=47 \\
\text { IG: } \mathrm{n}=69\end{array}$ & $\begin{array}{l}\text { IG: Patienten bestimmen } \\
\text { den Zeitpunkt der Wie- } \\
\text { deraufnahme der Nah- } \\
\text { rungszufuhr selbst; } \\
\text { KG: Serum Lipase muss } \\
\text { den Normalwert erreicht } \\
\text { haben, bevor die Nah- } \\
\text { rungsaufnahme gestar- } \\
\text { tet wird }\end{array}$ & $\begin{array}{l}\text { Schmerz- und Ernährungsprotokoll, durch- } \\
\text { schnittliche Zeit zwischen Entlassung und } \\
\text { oraler Nahrungsaufnahme, Länge der Kran- } \\
\text { kenhausverweildauer, tägliche Bestimmung } \\
\text { und Kontrolle über } 3 \text { Tage nach der ersten } \\
\text { Nahrungsaufnahme: Lipase, Amylase, Albu- } \\
\text { min, CRP, Blutzucker, Leukozytenanzahl }\end{array}$ & $\begin{array}{l}\text { durchschnittliche Zeit zwischen Entlassung und oraler } \\
\text { Nahrungsaufnahme: IG } 2 \text { Tage (IQR } 1-3) \text { vs. KG } 3 \text { Tage } \\
\text { (IQR 2-4) }(p<0,005) \text {; } \\
\text { vor und nach der ersten Mahlzeit, durchschnittliche } \\
\Delta \text { VAS: IG + } 3,14 \mathrm{~mm}( \pm 11,5 \mathrm{~mm}) \text { vs. KG }+2,85 \mathrm{~mm} \\
( \pm 16,4)(\mathrm{p}=0,597) \text {; } \\
\text { Länge der Krankenhausverweildauer: } \\
\text { IG } 7 \text { Tage (Median; IQR } 5-10,5) \text { vs. KG } 8 \text { Tage (Median; } \\
\text { IQR 5,75-12) }(\mathrm{p}=0,315)\end{array}$ \\
\hline $\begin{array}{l}\text { Sathiaraj et al. } \\
2008 \text { [13] }\end{array}$ & $\mathrm{Ib}$ & $\begin{array}{l}\text { randomisiert, } \\
\text { kontrolliert }\end{array}$ & $\begin{array}{l}101 \text { Patienten mit milder } \\
\text { akuter Pankreatitis } \\
\text { IG: } n=49 \\
\text { KG: } n=52\end{array}$ & $\begin{array}{l}\text { IG: weiche Nahrung; } \\
\text { KG: klare Flüssigkeiten }\end{array}$ & $\begin{array}{l}\text { Häufigkeit von Schmerzen, Dauer des Kran- } \\
\text { kenhausaufenthalts, Verträglichkeit der ora- } \\
\text { len Nahrungsaufnahme }\end{array}$ & $\begin{array}{l}\text { Deutlich kürzere Krankenausaufenthalte bei Patienten } \\
\text { der IG: durchschnittlich } 2 \text { Tage }(p<0,001) \text {. } \\
\text { Kein Unterschied zwischen den beiden Gruppen in der } \\
\text { Notwendigkeit die Diät zu beenden aufgrund von } \\
\text { Schmerzen. } \\
\text { Patienten der IG konsumierten deutlich mehr Kalorien } \\
\text { und Fett am ersten Studientag ( } p<0,001) \text {. }\end{array}$ \\
\hline
\end{tabular}

$C R P=C$-reaktives Protein, $I G=$ Interventionsgruppe, $I Q R=$ Interquartilbereich, $K G=$ Kontrollgruppe, $V A S=$ visuelle Analogskala 


\begin{tabular}{|c|c|c|c|c|c|c|c|}
\hline Referenz & Evidenzgrad & Studientyp & Teilnehmer/Studien & Intervention & Zielgröße & Hauptergebnis & Bemerkungen \\
\hline $\begin{array}{l}\text { Marik et al. } 2004 \\
\text { [14] }\end{array}$ & la & Metaanalyse & $\begin{array}{l}117 \text { Studien gefunden; } 6 \text { RCT } \\
\text { erfüllen alle Kriterien; } \\
263 \text { Patienten mit akuter } \\
\text { Pankreatitis } \\
\text { IG: } n=125 \\
\text { KG: } n=138\end{array}$ & $\begin{array}{l}\text { IG: enterale Ernährung über } \\
\text { eine nasojejunale Sonde; } \\
\text { KG: totale parenterale Ernäh- } \\
\text { rung }\end{array}$ & $\begin{array}{l}\text { Anzahl Infektionen, nicht infek- } \\
\text { tiöse Komplikationen, chirurgi- } \\
\text { sche Intervention, Länge des } \\
\text { Krankenhausaufenthalts, Kran- } \\
\text { kenhausmortalität }\end{array}$ & $\begin{array}{l}\text { EE } \leftrightarrow \text { TPE } \\
\text { Infektionen: RR 0,45 (95\%-KI } \\
0,26-0,78) \\
p=0,004 \\
\text { OP-Intervention: RR 0,48 } \\
(95 \%-K I 0,22-1,0) \\
p=0,05 \\
\text { Krankenhausaufenthalt: } \\
\text { - 2,9 Tage }(-1,6-4,3) \\
\text { p>0,001 } \\
\text { Mortalität: RR } 0,66 \text { ( } 95 \%-K I \\
0,32-1,37) \\
p=0,3 \\
\text { Komplikationen: RR 0,61 } \\
(95 \%-K I 0,31-1,22) \\
p=0,16\end{array}$ & $\begin{array}{l}\text { adäquat durchge- } \\
\text { führte Metaanalyse }\end{array}$ \\
\hline $\begin{array}{l}\text { Petrov et al. } 2008 \\
\text { [16] }\end{array}$ & la & Metaanalyse & $\begin{array}{l}11 \mathrm{RCT} \text { identifiziert; } 430 \mathrm{~Pa}- \\
\text { tienten mit akuter Pankreatitis } \\
\text { IG: } n=204 \\
\text { KG: } n=226\end{array}$ & $\begin{array}{l}\text { IG: enterale Ernährung über } \\
\text { eine nasojejunale Sonde; } \\
\text { KG: totale parenterale Ernäh- } \\
\text { rung }\end{array}$ & $\begin{array}{l}\text { infektiöse Komplikationen; } \\
\text { Mortalität }\end{array}$ & $\begin{array}{l}\text { EE } \leftrightarrow \text { TPE } \\
\text { Infektionen: RR 0,41 (95\%-KI } \\
0,30-0,57) \\
\text { p >0,001 } \\
\text { Mortalität: RR 0,60 (95\%-KI } \\
0,32-1,14) \\
p=0,12\end{array}$ & $\begin{array}{l}\text { adäquat durchge- } \\
\text { führte Metaanalyse }\end{array}$ \\
\hline $\begin{array}{l}\text { Al-Omran et al. } \\
2010[17]\end{array}$ & la & Metaanalyse & $\begin{array}{l}8 \text { RCT identifiziert; } \\
348 \text { Patienten mit akuter } \\
\text { Pankreatitis } \\
\text { IG: } n=164 \\
\text { KG: } n=184\end{array}$ & $\begin{array}{l}\text { IG: enterale Ernährung über } \\
\text { eine nasojejunale Sonde; } \\
\text { KG: totale parenterale Ernäh- } \\
\text { rung }\end{array}$ & $\begin{array}{l}\text { Mortalität, Multiorganversa- } \\
\text { gen, systemische Infektionen, } \\
\text { chirurgische Intervention, lo- } \\
\text { kale septische Komplikationen, } \\
\text { lokale nicht septische Kompli- } \\
\text { kationen, Länge des Kranken- } \\
\text { hausaufenthalts, Subgruppe: } \\
\text { Mortalität bei schwerer akuter } \\
\text { Pankreatitis }\end{array}$ & $\begin{array}{l}\text { EE } \leftrightarrow \text { TPE } \\
\text { Mortalität: RR 0,50 (95\%-KI } \\
0,28-0,91) \\
\text { MOF: RR 0,55 (95\%-KI 0,37- } \\
0,81 \text { ) } \\
\text { Infektionen: RR 0,39 (95\%-KI } \\
0,23-0,65) \\
\text { OP: RR 0,44 (95\%-KI 0,29-0,67) } \\
\text { lokale septische Komplikation } \\
\text { RR 0,74 (95\%-KI 0,40 - 1,35) } \\
\text { lokale Komplikation: RR 0,70 } \\
\text { (95\%-KI 0,43 - 1,13) } \\
\text { Krankenhausaufenthalt } \\
\text {-2,37 Tage in EN vs. TPE } \\
\text { (95\%-KI - 7,18-2,44) } \\
\text { Mortalität SAP: RR 0,18 (95\%-KI } \\
\text { 0,06-0,58) }\end{array}$ & $\begin{array}{l}\text { adäquat durchge- } \\
\text { führte Metaanalyse } \\
\text { nach Cochrane- } \\
\text { Standard }\end{array}$ \\
\hline
\end{tabular}




\begin{tabular}{|c|c|c|c|c|c|c|c|}
\hline Referenz & Evidenzgrad & Studientyp & Teilnehmer/Studien & Intervention & Zielgröße & Hauptergebnis & Bemerkungen \\
\hline $\begin{array}{l}\text { Modena et al. } \\
2006 \text { [44] }\end{array}$ & $\mathrm{Ib}$ & $\begin{array}{l}\text { randomisiert, } \\
\text { kontrolliert }\end{array}$ & $\begin{array}{l}87 \text { Patienten mit akuter } \\
\text { Pankreatitis } \\
\text { IG: } n=44 \\
\text { KG: } n=43\end{array}$ & $\begin{array}{l}\text { IG: enterale Ernährung über } \\
\text { eine nasojejunale Sonde (TEN } \\
\text { bei Patienten [ } n=44 \text { ] mit } \\
\text { schwerer NAP in der ersten } \\
\text { Behandlungswoche); } \\
\text { KG: totale parenterale Ernäh- } \\
\text { rung (TPN bei Patienten [ } n=43 \text { ] } \\
\text { mit schwerer NAP in der ersten } \\
\text { Behandlungswoche }\end{array}$ & $\begin{array}{l}\text { primär: infizierte Nekrose; } \\
\text { sekundär: Organversagen, } \\
\text { chirurgische Intervention, } \\
\text { Mortalität }\end{array}$ & $\begin{array}{l}\text { primär: } \\
\text { Infizierte Nekrose: IG } 20 \% \leftrightarrow \text { KG } \\
74 \% \\
\text { p }<0,001 \\
\text { sekundär: } \\
\text { Organversagen: IG } 31 \% \leftrightarrow \text { KG } \\
79 \% \\
\text { chirurgische Intervention: } \\
\text { IG } 25 \% \leftrightarrow \text { KG } 88 \% \\
\text { p }<0,001 \\
\text { Mortalität: IG } 5 \% \leftrightarrow \text { KG } 35 \% \\
\text { p }<0,001\end{array}$ & $\begin{array}{l}\text { langer Rekrutie- } \\
\text { rungszeitraum von } \\
5 \text { Jahren; adäquat } \\
\text { vergleichbare Pa- } \\
\text { tientengruppen mit } \\
\text { schwerer akuter } \\
\text { Pankreatitis bei Ein- } \\
\text { schluss }\end{array}$ \\
\hline
\end{tabular}

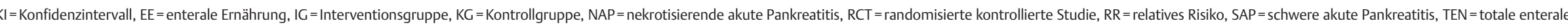
Ernährung, TPE = totale parenterale Ernährung

Evidenztabelle 3 Durchführung der enteralen Ernährung bei akuter Pankreatitis.

\begin{tabular}{|c|c|c|c|c|c|c|c|}
\hline Referenz & Evidenzgrad & Studientyp & Teilnehmer & Intervention & Zielgröße & Hauptergebnis & Bemerkungen \\
\hline $\begin{array}{l}\text { Petrov et al. } 2009 \\
\text { [20] }\end{array}$ & la & Metaanalyse & $\begin{array}{l}\text { 20 RCT identifiziert; } \\
1070 \text { Patienten } \\
\text { IG: } n=529 \\
\text { KG: } n=541\end{array}$ & $\begin{array}{l}\text { IG: enterale Ernährung: Semi- } \\
\text { Elementardiät, hochmolekula- } \\
\text { re Diät oder angereichert mit } \\
\text { Ballaststoffen, Probiotika oder } \\
\text { Immunonutritions; } \\
\text { KG: parenterale Ernährung }\end{array}$ & $\begin{array}{l}\text { Toleranz und Sicherheit der en- } \\
\text { teralen Ernährung bei Patien- } \\
\text { ten mit akuter Pankreatitis } \\
\text { (Nahrungsintoleranz, infektiö- } \\
\text { se Komplikationen, Mortalität) }\end{array}$ & $\begin{array}{l}\text { keine der folgenden Interven- } \\
\text { tionen wurde mit einem deut- } \\
\text { lichen Unterschied der Nah- } \\
\text { rungstoleranz assoziiert; das } \\
\text { Risiko für infektiöse Komplika- } \\
\text { tionen und die Mortalität zeig- } \\
\text { ten keinen bedeutenden Unter- } \\
\text { schied in den verschiedenen } \\
\text { Vergleichsgruppen }\end{array}$ & $\begin{array}{l}\text { indirekte Metaana- } \\
\text { lyse, da unter- } \\
\text { schiedliche Ver- } \\
\text { gleichsgruppen }\end{array}$ \\
\hline $\begin{array}{l}\text { Tiengou et al. } \\
2006 \text { [19] }\end{array}$ & Ib & $\begin{array}{l}\text { randomisiert, } \\
\text { kontrolliert }\end{array}$ & $\begin{array}{l}\text { 30 Patienten mit akuter } \\
\text { Pankreatitis } \\
\text { IG: } \mathrm{n}=15 \\
\text { KG: } \mathrm{n}=15\end{array}$ & $\begin{array}{l}\text { IG: enterale Ernährung über } \\
\text { eine nasojejunale Sonde: Semi- } \\
\text { Elementardiät ( } 35 \mathrm{kcal} / \mathrm{kg} \mathrm{KG/} \\
\text { d); } \\
\text { KG: enterale Ernährung über } \\
\text { eine nasojejunale Sonde: hoch- } \\
\text { molekulare Diät }\end{array}$ & $\begin{array}{l}\text { Gewichtsverlust, Dauer des } \\
\text { Krankenhausaufenthalts, Infek- } \\
\text { tionsrate }\end{array}$ & $\begin{array}{l}\text { Dauer des Krankenhausaufent- } \\
\text { halts: } \\
\text { bei der IG war der Aufenthalt } \\
\text { kürzer (IG } 23 \pm 2 \text { Tage vs. KG } \\
27 \pm 1 \text { Tag, } p=0,006) \\
\text { Gewichtsverlust: } \\
\text { Bei der IG war der Gewichts- } \\
\text { verlust geringer (IG }-1,3 \pm \\
1,1 \mathrm{~kg} \text { vs. KG }-2,4 \pm 0 \mathrm{~kg} \text {, } \\
\mathrm{p}=0,01 \text { ) } \\
\text { Infektionsrate: } \\
\text { IG: } 1 \text { Patient vs. KG: } 3 \text { Patienten }\end{array}$ & $\begin{array}{l}\text { eingeschränkte } \\
\text { Aussagekraft bei } \\
\text { geringer Fallzahl }\end{array}$ \\
\hline
\end{tabular}




\begin{tabular}{|c|c|c|c|c|c|c|c|}
\hline \multicolumn{8}{|c|}{ Evidenztabelle 3 (Fortsetzung) } \\
\hline Referenz & Evidenzgrad & Studientyp & Teilnehmer & Intervention & Zielgröße & Hauptergebnis & Bemerkungen \\
\hline $\begin{array}{l}\text { Petrov et al. } \\
\text { 2008a [23] }\end{array}$ & la & Metaanalyse & $\begin{array}{l}78 \text { Patienten aus } 3 \text { RCT } \\
\text { IG: } n=40 \\
\text { KG: } n=38\end{array}$ & $\begin{array}{l}\text { IG: enterale Ernährung mit } \\
\text { Immunonutritions } \\
\text { KG: standard enterale } \\
\text { Ernährung }\end{array}$ & $\begin{array}{l}\text { infektiöse Komplikationen, } \\
\text { Mortalität im Krankenhaus, } \\
\text { Dauer des Krankenhausauf- } \\
\text { enthalts }\end{array}$ & $\begin{array}{l}\text { IG vs. KG: } \\
\text { keine deutliche Senkung des } \\
\text { Risikos für infektiöse Komplika- } \\
\text { tionen (RR 0,82; } 95 \% \text {-KI 0,44- } \\
1,53 \text { [p }=0,53] \text { ); } \\
\text { kein signifikanter Unterschied } \\
\text { im Mortalitätsrisiko (RR 0,64; } \\
95 \%-K I 0,20-2,07 \text { [ } p=0,46] \text { ); } \\
\text { kein signifikanter Unterschied } \\
\text { bei der Dauer des Kranken- } \\
\text { hausaufenthalts }(p=0,80)\end{array}$ & \\
\hline $\begin{array}{l}\text { Oláh et al. } 2007 \\
\text { [46] }\end{array}$ & $\mathrm{Ib}$ & $\begin{array}{l}\text { randomisiert, } \\
\text { kontrolliert } \\
\text { (doppelblind) }\end{array}$ & $\begin{array}{l}70 \text { Patienten mit schwerer } \\
\text { akuter Pankreatitis }\end{array}$ & $\begin{array}{l}\text { IG: nasojejunale Ernährung } \\
\text { und jeweils } 4 \text { unterschiedliche } \\
\text { Lactobacilli-Präparate mit } 10^{10} \\
\text { CFU und zusätzlich Präbiotika } \\
\text { (Inulin, beta-Glukan, resistente } \\
\text { Stärke und Pektin) } \\
\text { KG: nasojejunale Ernährung } \\
\text { und Präbiotika }\end{array}$ & $\begin{array}{l}\text { Multiorganversagen, septische } \\
\text { Komplikationen, Mortalität, } \\
\text { SIRS }\end{array}$ & $\begin{array}{l}\text { IG: geringere Inzidenz an MOF, } \\
\text { septische Komplikationen und } \\
\text { Mortalität, aber keine signifi- } \\
\text { kanten Unterschiede; } \\
\text { die Gesamtinzidenz von SIRS } \\
\text { und MOF war signifikant unter- } \\
\text { schiedlich (IG } 8 \text { vs. KG 14; } \\
\text { p<0,05); } \\
\text { die Zahl der Patienten mit wie- } \\
\text { derauftretenden Komplikatio- } \\
\text { nen war signifikant geringer in } \\
\text { der IG verglichen mit der KG } \\
\text { (p<0,05); } \\
\text { geringere Raten von spätem } \\
\text { MOF (nach } 48 \text { Stunden) in IG } \\
\text { (IG } 3,0 \% \text { vs. KG } 17,2 \% \text { ) }\end{array}$ & \\
\hline $\begin{array}{l}\text { Besselink et al. } \\
2008 \text { [22] }\end{array}$ & $\mathrm{Ib}$ & $\begin{array}{l}\text { randomisiert, } \\
\text { kontrolliert }\end{array}$ & $\begin{array}{l}296 \text { Patienten mit prognosti- } \\
\text { zierter starker akuter Pankrea- } \\
\text { titis } \\
\text { IG: } n=152 \\
\text { KG: } n=144\end{array}$ & $\begin{array}{l}\text { IG: Probiotikum-Misch-Präpa- } \\
\text { rat (Multispezies); enterale } \\
\text { Einnahme täglich 2-mal für } \\
28 \text { Tage } \\
\text { KG: Placebo; enterale Einnah- } \\
\text { me täglich 2-mal für } 28 \text { Tage }\end{array}$ & $\begin{array}{l}\text { infektiöse Komplikationen: } \\
\text { Entzündung des Pankreas, } \\
\text { Nekrose, Bakteriämie, Pneu- } \\
\text { monie, Urosepsis, infizierte } \\
\text { Aszites während der Einnahme } \\
\text { und } 90 \text { Tage danach; Mortalität }\end{array}$ & $\begin{array}{l}\text { infektiöse Komplikationen: } \\
\text { IG: } n=46(30 \%) \text { der Patienten } \\
\text { vs. KG: } n=41(28 \%) \text { (RR 1,06; } \\
95 \%-K I ~ 0,75-1,51) \text {; } \\
\text { Mortalität: } \\
\text { IG: } n=24(16 \%) \text { vs. KG } n=9 \\
(6 \%)(\text { RR 2,53; } 95 \%-K I 1,22- \\
5,25) ; \\
\text { Darm-Ischämie: } \\
\text { IG: } n=9 \text { Patienten (8 mit fata- } \\
\text { len Folgen } \rightarrow \text { Tod) vs. KG: keine } \\
(p=0,004)\end{array}$ & $\begin{array}{l}\text { adäquate Fallzahl; } \\
\text { Hauptkomplikation } \\
\text { Darmischämie in } \\
\text { der IG, ggf. durch } \\
\text { Kombination aus } \\
\text { Prä- und Probiotika }\end{array}$ \\
\hline $\begin{array}{l}\text { Jiang et al. } 2007 \\
\text { [26] }\end{array}$ & la & Metaanalyse & $\begin{array}{l}3 \text { RTC; } \\
131 \text { Patienten mit starker aku- } \\
\text { ten Pankreatitis } \\
\text { IG: } n=61 \\
\text { KG: } n=64 \text {, davon } 38 \text { mit NJEN } \\
\text { und } 26 \text { mit TPN }\end{array}$ & $\begin{array}{l}\text { IG: frühe enterale Ernährung } \\
\text { über eine nasogastrale Sonde } \\
\text { KG: enterale Ernährung über } \\
\text { eine NJEN oder TPE }\end{array}$ & $\begin{array}{l}\text { Mortalität, Dauer des Kranken- } \\
\text { hausaufenthalts, MOF (Versa- } \\
\text { gen von mind. } 2 \text { Organen), } \\
\text { Schmerzen verursacht durch } \\
\text { Wiederaufnahme der Nah- } \\
\text { rungszufuhr, Komplikationen } \\
\text { systematischer oder lokaler } \\
\text { Infektionen }\end{array}$ & $\begin{array}{l}\text { keine signifikanten Unterschie- } \\
\text { de zwischen den Gruppen bei } \\
\text { Mortalität, Dauer des Kranken- } \\
\text { hausaufenthalts, Rate der } \\
\text { Komplikationen bei Infektio- } \\
\text { nen, MOF, Einstufungsrate } \\
\text { nach ICU, Schmerzen verur- } \\
\text { sacht durch Wiederaufnahme } \\
\text { der Nahrungszufuhr }\end{array}$ & \\
\hline
\end{tabular}




\begin{tabular}{|c|c|c|c|c|c|c|c|}
\hline Referenz & Evidenzgrad & Studientyp & Teilnehmer & Intervention & Zielgröße & Hauptergebnis & Bemerkungen \\
\hline $\begin{array}{l}\text { Petrov et al. } 2008 \\
\text { [25] }\end{array}$ & la & Metaanalyse & $\begin{array}{l}4 \mathrm{RCT} \text {; } \\
92 \text { Patienten mit starker akuter } \\
\text { Pankreatitis } \\
\text { IG: } \mathrm{n}=43 \\
\text { KG: } \mathrm{n}=36\end{array}$ & $\begin{array}{l}\text { IG: enterale Ernährung über } \\
\text { eine nasogastrale Sonde; } \\
\text { KG: enterale Ernährung über } \\
\text { eine nasojejunale Sonde }\end{array}$ & $\begin{array}{l}\text { Mortalität, Schmerzen verur- } \\
\text { sacht durch Wiederaufnahme } \\
\text { der Nahrungszufuhr }\end{array}$ & $\begin{array}{l}\text { IG vs. KG: } \\
\text { Mortalität: RR 0,77; } 95 \%-K I \\
0,37-1,62(p=0,50) \text {; } \\
\text { Intoleranz der Nahrungsauf- } \\
\text { nahme: RR } 1,09 ; 95 \%-K I \\
0,46-2,59(p=0,84)\end{array}$ & \\
\hline $\begin{array}{l}\text { Makola et al. } 2006 \\
\text { [45] }\end{array}$ & III & $\begin{array}{l}\text { Beobachtungs- } \\
\text { studie }\end{array}$ & 126 Patienten & $\begin{array}{l}\text { enterale Ernährung via PEG-J } \\
\text { und hochmolekulare Sonden- } \\
\text { kost }\end{array}$ & $\begin{array}{l}\text { Anzeichen der Verbesserung im } \\
\text { CT Severity Index, Dauer der } \\
\text { enteralen Ernährung, Dauer } \\
\text { des Krankenhausaufenthaltes, } \\
\text { Gewichtsveränderung, Serum- } \\
\text { Albumin }\end{array}$ & $\begin{array}{l}\text { Dauer der enteralen Ernäh- } \\
\text { rung: } 18,9(2,4-111,7) \text { Wo- } \\
\text { chen; Median CT Severity In- } \\
\text { dex: Senkung von } 4 \text { auf } 2 \text { ( } p< \\
\text { 0,001); Gewichtsveränderung: } \\
\text { untergewichtige Patienten } \rightarrow \\
\text { Zunahme von } 9,8 \text { Pfund; über- } \\
\text { gewichtige und adipöse Patien- } \\
\text { ten } \rightarrow \text { Abnahme von } 7,2 \text { und } \\
28,8 \text { Pfund; Serum-Albumin: } \\
\text { Anstieg von } 3 \text { auf } 3,8 \mathrm{~g} / \mathrm{dL} \\
(p<0,001)\end{array}$ & \\
\hline
\end{tabular}

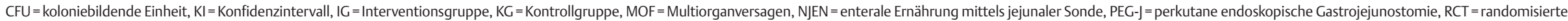
kontrollierte Studie, RR= relatives Risiko, SIRS= systemisches inflammatorisches Response-Syndrom, TPE = totale parenterale Ernährung

Evidenztabelle 4 Durchführung der parenteralen Ernährung bei akuter Pankreatitis.

\begin{tabular}{|c|c|c|c|c|c|c|c|}
\hline Referenz & Evidenzgrad & Studientyp & Teilnehmer & Intervention & Zielgröße & Hauptergebnis & Bemerkungen \\
\hline $\begin{array}{l}\text { Siriwardena et al. } \\
2007 \text { [24] }\end{array}$ & $\mathrm{Ib}$ & $\begin{array}{l}\text { randomisiert, } \\
\text { kontrolliert }\end{array}$ & $\begin{array}{l}43 \text { Patienten mit prognosti- } \\
\text { zierter starker akuter Pankrea- } \\
\text { titis } \\
\text { IG: } n=22 \\
\text { KG: } n=21\end{array}$ & $\begin{array}{l}\text { IG: maximale konventionelle } \\
\text { Therapie plus intravenöse an- } \\
\text { tioxidative Therapie für } 7 \text { Tage; } \\
\text { KG: maximale konventionelle } \\
\text { Therapie plus intravenös ver- } \\
\text { abreichtes Placebo für } 7 \text { Tage }\end{array}$ & Organversagen & $\begin{array}{l}\text { relative Serumlevel der Anti- } \\
\text { oxidanzien stiegen in IG, wäh- } \\
\text { rend Marker für oxidativen Stress } \\
\text { abnahmen; } \\
\text { Organversagen: } \\
\text { IG vs. KG: } 32 \text { vs. } 17 \%(p=0,33)\end{array}$ & $\begin{array}{l}\text { eingeschränkte } \\
\text { Aussagekraft bei } \\
\text { geringer Fallzahl }\end{array}$ \\
\hline $\begin{array}{l}\text { Bansal et al. } 2011 \\
\text { [29] }\end{array}$ & $\mathrm{Ib}$ & $\begin{array}{l}\text { randomisiert, } \\
\text { kontrolliert }\end{array}$ & $\begin{array}{l}39 \text { Patienten mit starker akuter } \\
\text { Pankreatitis } \\
\text { IG: } n=19 \\
\text { KG: } n=20\end{array}$ & $\begin{array}{l}\text { IG: Standard-Behandlung + } \\
\text { Antioxidanzien (Vitamin A, } \\
\text { Vitamin E, Vitamin C) für einen } \\
\text { Zeitraum von } 14 \text { Tagen; } \\
\text { KG: Standardbehandlung }\end{array}$ & $\begin{array}{l}\text { Organversagen am Tag 7, Dau- } \\
\text { er des Krankenhausaufent- } \\
\text { halts, Multiorgandysfunktion } \\
\text { am 7. Tag, Mortalität, Verän- } \\
\text { derungen der Marker für oxi- } \\
\text { dativen Stress }\end{array}$ & $\begin{array}{l}\text { kein signifikanter Unterschied } \\
\text { zw. den beiden Gruppen bei Or- } \\
\text { ganversagen }(p=1,0) \text {, Multior- } \\
\text { gandysfunktion ( } p=0,8) \text {, Dauer } \\
\text { des Krankenhausaufenthalts } \\
\text { ( } p=0,29) \text {; } \\
\text { Mortalität: IG: alle überlebten; } \\
\text { KG: } 2 \text { Patienten starben; } \\
\text { Veränderungen der Level von } \\
\text { Malondialdehyd, Superoxiddis- } \\
\text { mutase und reduzierte Gluta- } \\
\text { thione waren am } 7 \text {. Tag in den } \\
\text { beiden Gruppen nicht signifikant } \\
\text { unterschiedlich }\end{array}$ & $\begin{array}{l}\text { eingeschränkte } \\
\text { Aussagekraft bei } \\
\text { geringer Fallzahl }\end{array}$ \\
\hline
\end{tabular}


Evidenztabelle 5 Ernährung bei chronischer Pankreatitis.

\begin{tabular}{|c|c|c|c|c|c|c|}
\hline Referenz & $\begin{array}{l}\text { Evidenz- } \\
\text { grad }\end{array}$ & Studientyp & Teilnehmer & Intervention & Zielgröße & Hauptergebnis \\
\hline $\begin{array}{l}\text { Stanga et al. } \\
2005 \text { [36] }\end{array}$ & III & $\begin{array}{l}\text { Beobach- } \\
\text { tungsstudie }\end{array}$ & $\begin{array}{l}57 \text { Patienten } \\
\text { mit chronischer } \\
\text { Pankreatitis }\end{array}$ & $\begin{array}{l}\text { Erhalt der enteralen } \\
\text { Ernährung durch PEG/ } \\
\text { J ( } n=53) \text {; Erhalt der } \\
\text { enteralen Ernährung } \\
\text { durch DPEJ }(n=4)\end{array}$ & $\begin{array}{l}\text { Gewichtsverände- } \\
\text { rung, abdominale } \\
\text { Schmerzen, Grad der } \\
\text { Mangelernährung }\end{array}$ & $\begin{array}{l}\text { Gewichtsveränderung: } \\
\text { das durchschnittliche Gewicht stieg } \\
\text { deutlich von } 64,8 \mathrm{~kg} \text { am Tag } 1 \text { auf } \\
69,1 \mathrm{~kg} \text { am Tag } 180 \text { an ( } p=0,001) ; \\
\text { abdominale Schmerzen: } \\
\text { Senkung von } 96 \text { auf } 23 \% \text {; } \\
\text { Mangelernährung: } \\
\text { Senkung des Grades der Mangelernäh- } \\
\text { rung }\end{array}$ \\
\hline $\begin{array}{l}\text { Skipworth } \\
\text { et al. } 2011 \\
{[37]}\end{array}$ & III & $\begin{array}{l}\text { Beobach- } \\
\text { tungsstudie }\end{array}$ & $\begin{array}{l}58 \text { Patienten } \\
\text { mit chronischer } \\
\text { Pankreatitis }\end{array}$ & $\begin{array}{l}\text { enterale Ernährung } \\
\text { über eine nasojejuna- } \\
\text { le Sonde }\end{array}$ & $\begin{array}{l}\text { Gewichtsverände- } \\
\text { rung, schmerzlindern- } \\
\text { der Effekt, Toleranz } \\
\text { und Komplikationen, } \\
\text { Pankreas-Morpholo- } \\
\text { gie, Bluttest }\end{array}$ & 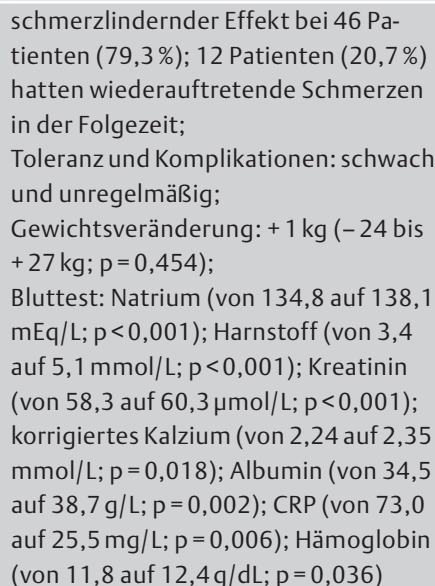 \\
\hline
\end{tabular}

$C R P=C$-reaktives Protein, DPE $=$ direkte perkutane endoskopische Jejunostomie, $\mathrm{PEG} / \mathrm{J}=$ perkutane endoskopische Gastrojejunostomie

\section{Literatur}

1 Lübke H, Meier R, Lochs $H$ et al. DGEM-Leitlinie Enterale Ernährung: Gastroenterologie. Aktuel Ernahrungsmed 2003; 28 (Suppl. 01): S69-S86

2 Schulz R, Bischoff S, Koletzko B. Leitlinie Parenterale Ernährung der DGEM: Gastroenterologie. Aktuel Ernahrungsmed 2007; 32 (Suppl. 01): $S 93-S 96$

3 Meier R, Ockenga J, Pertkiewicz $M$ et al. ESPEN Guidelines on Enteral Nutrition: Pancreas. Clin Nutr 2006; 25: 275-284

4 Gianotti L, Meier R, Lobo DN et al. ESPEN Guidelines on Parenteral Nutrition: pancreas. Clin Nutr 2009; 28: 428-435

5 Stingel $K$, Schütz T, Koller $M$ et al. Leitlinie der Deutschen Gesellschaft für Ernährungsmedizin (DGEM) - Methodik zum Leitlinien-Update Klinische Ernährung. Aktuel Ernahrungsmed 2013; 38: 90-96

6 Koller M, Schutz T, Valentini L et al. Outcome models in clinical studies: Implications for designing and evaluating trials in clinical nutrition. Clin Nutr 2013; 32: 650-657

7 Dickerson RN, Vehe KL, Mullen JL et al. Resting energy expenditure in patients with pancreatitis. Crit Care Med 1991; 19: 484-490

8 Shaw JH, Wolfe RR. Glucose, fatty acid, and urea kinetics in patients with severe pancreatitis. The response to substrate infusion and total parenteral nutrition. Ann Surg 1986; 204: 665-672

9 Pirlich M, Schwenk A, Müller M. DGEM-Leitlinie Enterale Ernährung: Ernährungsstatus. Aktuel Ernahrungsmed 2003; 28 (Suppl. 01): S10S25

10 Jacobson $B C$, Vander Vliet MB, Hughes MD et al. A prospective, randomized trial of clear liquids versus low-fat solid diet as the initial meal in mild acute pancreatitis. Clin Gastroenterol Hepatol 2007; 5: 946 - 951; quiz 886

11 Moraes JM, Felga GE, Chebli LA et al. A full solid diet as the initial meal in mild acute pancreatitis is safe and result in a shorter length of hospitalization: results from a prospective, randomized, controlled, doubleblind clinical trial. J Clin Gastroenterol 2010; 44: 517-522

12 Teich N, Aghdassi A, Fischer J et al. Optimal timing of oral refeeding in mild acute pancreatitis: results of an open randomized multicenter trial. Pancreas 2010; 39: 1088-1092
13 Sathiaraj E, Murthy S, Mansard MJ et al. Clinical trial: oral feeding with a soft diet compared with clear liquid diet as initial meal in mild acute pancreatitis. Aliment Pharmacol Ther 2008; 28: 777-781

14 Marik PE, Zaloga GP. Meta-analysis of parenteral nutrition versus enteral nutrition in patients with acute pancreatitis. BMJ 2004; 328 : 1407

15 McClave SA, Chang WK, Dhaliwal $R$ et al. Nutrition support in acute pancreatitis: a systematic review of the literature. JPEN J Parenter Enteral Nutr 2006; 30: $143-156$

16 Petrov MS, Pylypchuk RD, Emelyanov NV. Systematic review: nutritional support in acute pancreatitis. Aliment Pharmacol Ther 2008; 28: 704-712

17 Al-Omran M, Albalawi ZH, Tashkandi MF et al. Enteral versus parenteral nutrition for acute pancreatitis. Cochrane Database Syst Rev2010: (1): CD002837. doi: CD002837

$18 \mathrm{LiJ}-\mathrm{Y}$, Yu T, Chen G-C et al. Enteral Nutrition within 48 Hours of Admission Improves Clinical Outcomes of Acute Pancreatitis by Reducing Complications: A Meta-Analysis. PLoS ONE 2013; 8: e64926

19 Tiengou LE, Gloro R, Pouzoulet J et al. Semi-elemental formula or polymeric formula: is there a better choice for enteral nutrition in acute pancreatitis? Randomized comparative study. JPEN J Parenter Enteral Nutr 2006; 30: $1-5$

20 Petrov MS, Loveday BP, Pylypchuk RD et al. Systematic review and metaanalysis of enteral nutrition formulations in acute pancreatitis. $\mathrm{Br} \mathrm{J}$ Surg 2009; 96: $1243-1252$

21 Sun S, Yang K, He X et al. Probiotics in patients with severe acute pancreatitis: a meta-analysis. Langenbecks Arch Surg 2009; 394: 171 - 177

22 Besselink MG, van Santvoort HC, Buskens E et al. Probiotic prophylaxis in predicted severe acute pancreatitis: a randomised, double-blind, placebo-controlled trial. Lancet 2008; 371: 651-659

23 Petrov MS, Atduev VA, Zagainov VE. Advanced enteral therapy in acute pancreatitis: is there a room for immunonutrition? A meta-analysis. Int J Surg 2008; 6: 119-124

24 Siriwardena AK, Mason JM, Balachandra S et al. Randomised, double blind, placebo controlled trial of intravenous antioxidant (n-acetylcysteine, selenium, vitamin C) therapy in severe acute pancreatitis. Gut 2007; 56 : $1439-1444$ 
25 Petrov MS, Correia MI, Windsor JA. Nasogastric tube feeding in predicted severe acute pancreatitis. A systematic review of the literature to determine safety and tolerance. JOP 2008; 9: 440-448

26 Jiang $K$, Chen XZ, Xia $Q$ et al. Early nasogastric enteral nutrition for severe acute pancreatitis: a systematic review. World J Gastroenterol 2007; $13: 5253-5260$

27 Lasztity N, Hamvas J, Biro L et al. Effect of enterally administered n-3 polyunsaturated fatty acids in acute pancreatitis - a prospective randomized clinical trial. Clin Nutr 2005; 24: 198 -205

28 Asrani V, Chang WK, Dong $Z$ et al. Glutamine supplementation in acute pancreatitis: a meta-analysis of randomized controlled trials. Pancreatology 2013; 13: 468-474

29 Bansal D, Bhalla A, Bhasin DK et al. Safety and efficacy of vitamin-based antioxidant therapy in patients with severe acute pancreatitis: a randomized controlled trial. Saudi J Gastroenterol 2011; 17: 174-179

30 Ockenga J. Importance of nutritional management in diseases with exocrine pancreatic insufficiency. HPB (Oxford) 2009; 11 (Suppl. 03): $11-15$

31 Armbrecht U. Chronic pancreatitis: weight loss and poor physical performance - experience from a specialized rehabilitation centre. Rehabilitation (Stuttg) 2001; 40: 332-336

32 Riediger $H$, Adam $U$, Fischer $E$ et al. Long-term outcome after resection for chronic pancreatitis in 224 patients. J Gastrointest Surg 2007; 11: 949-959; discussion 959-960

33 Haaber $A B$, Rosenfalck $A M$, Hansen $B$ et al. Bone mineral metabolism, bone mineral density, and body composition in patients with chronic pancreatitis and pancreatic exocrine insufficiency. Int J Pancreatol 2000; $27: 21-27$

34 Fitzsimmons D, Kahl S, Butturini $G$ et al. Symptoms and quality of life in chronic pancreatitis assessed by structured interview and the EORTC QLQ-C30 and QLQ-PAN26. Am J Gastroenterol 2005; 100: 918-926

35 Singh S, Midha S, Singh $N$ et al. Dietary counseling versus dietary supplements for malnutrition in chronic pancreatitis: a randomized controlled trial. Clin Gastroenterol Hepatol 2008; 6: 353 - 359
36 Stanga Z, Giger U, Marx A et al. Effect of jejunal long-term feeding in chronic pancreatitis. JPEN J Parenter Enteral Nutr 2005; 29: 12-20

37 Skipworth JR, Raptis DA, Wijesuriya S et al. The use of nasojejunal nutrition in patients with chronic pancreatitis. JOP 2011; 12: 574-580

38 Chronic Pancreatitis German Society of Digestive and Metabolic Diseases (DGVS). Hoffmeister A, Mayerle J, Beglinger C et al. S3-Consensus guidelines on definition, etiology, diagnosis and medical, endoscopic and surgical management of chronic pancreatitis German Society of Digestive and Metabolic Diseases (DGVS). Z Gastroenterol 2012; 50: $1176-1224$

39 Dominguez-Munoz JE. Pancreatic enzyme therapy for pancreatic exocrine insufficiency. Curr Gastroenterol Rep 2007; 9: 116-122

40 Dominguez-Munoz JE, Iglesias-Garcia J. Oral pancreatic enzyme substitution therapy in chronic pancreatitis: is clinical response an appropriate marker for evaluation of therapeutic efficacy? JOP 2010; 11: $158-162$

41 Dominguez-Munoz JE. Pancreatic exocrine insufficiency: diagnosis and treatment. J Gastroenterol Hepatol 2011; 26 (Suppl. 02): 12-16

42 Hartl WH, Parhofer KG, Kuppinger D et al. und das DGEM Steering Committee. Leitlinie der Deutschen Gesellschaft für Ernährungsmedizin (DGEM) in Zusammenarbeit mit der GESKES und der AKE: Besonderheiten der Überwachung bei künstlicher Ernährung - Teil des laufenden S3-Leitlinienprojekts Klinische Ernährung. Aktuel Ernahrungsmed 2013; 38: e90-e100

43 Braganza JM, Lee SH, McCloy RF et al. Chronic pancreatitis. Lancet 2011; 377: $1184-1197$

44 Targarona Modena J, Barreda Cevasco L, Arroyo Basto C et al. Total enteral nutrition as prophylactic therapy for pancreatic necrosis infection in severe acute pancreatitis. Pancreatology 2006; 6: 58-64

45 Makola D, Krenitsky J, Parrish C et al. Efficacy of enteral nutrition for the treatment of pancreatitis using standard enteral formula. Am J Gastroenterol 2006; 101: 2347-2355

46 Olah A, Belagyi T, Poto $L$ et al. Synbiotic control of inflammation and infection in severe acute pancreatitis: a prospective, randomized, double blind study. Hepatogastroenterology 2007; 54: 590-594 